$\angle$ Research Square
Preprints are preliminary reports that have not undergone peer review. They should not be considered conclusive, used to inform clinical practice, or referenced by the media as validated information.

\title{
Comparison of clinical characteristics, efficacy and survival of newly diagnosed extramedullary multiple myeloma patients between single and multiple sites invasion
}

\section{Yongfeng Zhao}

Wuhan University Zhongnan Hospital

Fuling Zhou ( $\nabla$ zhoufuling@whu.edu.cn )

Wuhan University Zhongnan Hospital https://orcid.org/0000-0003-0982-0382

\section{Research Article}

Keywords: Multiple myeloma, Proteasome inhibitors, Immunomodulators, Overall survival, Extramedullary extraosseous, Progression free survival

Posted Date: November 5th, 2021

DOI: https://doi.org/10.21203/rs.3.rs-1033633/v1

License: (우 (i) This work is licensed under a Creative Commons Attribution 4.0 International License. Read Full License 


\section{Abstract}

\section{Purpose}

We aimed to compare the clinical characteristics, efficacy and survival of newly diagnosed extramedullary multiple myeloma patients (EMM) between single and multiple sites invasion.

\section{Methods}

A total of $90 \mathrm{EMM}$ patients were included. The characteristics including gender, age, Durie-Salmon stage, ISS stage, hemoglobin, blood calcium, creatinine, M-protein types, $\beta 2$-microglobulin, lactate dehydrogenase and so on were analyzed. We compared the overall remission rates (ORR) in patients with single site invasion and multiple sites invasion. Progression free survival (PFS) and overall survival (OS) were also compared.

\section{Results}

Patients with multiple sites invasion had higher lactate dehydrogenase than single site invasion $(179.0 \mathrm{U} / \mathrm{L}$ vs. $154.7 \mathrm{U} / \mathrm{L}, P=0.016)$. The ORR in patients with single site invasion $(72.1 \%)$ was not significantly higher than multiple sites invasion (68.2\%) $(P=0.690)$. In patients with multiple sites invasion, PI-based regimen $(78.9 \% \mathrm{vs.}$ $33.3 \%, P=0.035)$ or $\mathrm{PI}$ combined with IMiD regimen ( $84.6 \%$ vs. $33.3 \%, P=0.026)$ could achieve superior efficacy than routine chemotherapy. Among patients with single site invasion, the COX model analysis showed that proteasome inhibitors combined with immunomodulators could significantly improve the PFS (HR=0.080, $95 \% \mathrm{Cl}: 0.007-0.855, P=0.037)$. Among patients with multiple sites invasion, the associations of RISS 3 with poor PFS (HR=4.081, 95\%Cl: 1.533-10.865, $P=0.005)$ and OS (HR=13.295, 95\%Cl: 3.219-54.907, $P=0.000)$ were showed.

\section{Conclusion}

RISS stage 3 was possibly associated with poor survival of extramedullary multiple myeloma patients with multiple sites invasion. We propose a prospective and large-sample study to explore the effects of new drugs and autologous hematopoietic stem cell transplantation on survival of patients at RISS stage 3 .

\section{Introduction}

Multiple myeloma (MM) is the second most common hematological malignancy characterized by clonal plasma cell infiltration in bone marrow and the presence of monoclonal immunoglobulins in blood and/or urine, that cause a series of clinical symptoms, such as anemia, hypercalcemia, renal damage, and bone destruction (Kumar SK et al. 2017). Most of tumor cells are confined to the bone marrow, and in some patients, malignant plasma cells can break through the bone marrow and bone tissue involving the periosteal tissue, forming tumorous masses in the adjacent bone sites. In addition, the malignant plasma cells can also enter the blood circulation and colonize the distant tissue to form tumorous masses. These two types of multiple myeloma are known as extramedullary multiple myeloma (EMM), the former was called extramedullary-bone related (EMB), the latter was called extramedullary extraosseous (EME) (Bhutani M et al. 2020; Jagosky MH and Usmani SZ 2020; Montefusco V et al. 2019). Until now, extramedullary multiple myeloma is inherently a high-risk stage disease with a poor prognosis, and there are limited data regarding the 
basic features of it. EMM can be present at initial diagnosis with an estimated incidence of $7 \%$ or at relapse with an estimated incidence of $6 \%$ (Varettoni M et al. 2010), with the overall incidence of $18.2 \%$ (Gagelmann $N$ et al. 2018). The patients possibly had single site invasion, possibly had two or more sites invasion. Multiple sites invasion were reported in 52\% of EMM patients, and the most commonly involved sites were soft tissue and skin, pleura, lung, lymph nodes, liver, etc (Avivi I et al. 2019). So far, there were few studies about patients with single or multiple sites invasion. The clinical characteristics, efficacy, and the survival of extramedullary multiple myeloma patients with single or multiple sites invasion remained uncertain. Therefore, the purpose of this study was to compare the clinical characteristics, efficacy, and the survival of patients with single site invasion and multiple sites invasion.

\section{Methods}

\section{Study Design and Participants}

A total of 90 extramedullary multiple myeloma patients who were newly diagnosed according to the International Myeloma Working Group (IMWG) diagnostic criteria ((Rajkumar SV et al. 2014) in Zhongnan Hospital of Wuhan University from January 2013 to December 2020 were included. Extramedullary multiple myeloma was defined as plasmacytoma involving tissues other than bone marrow and bone. All patients were hospitalized during the initial diagnosis and treatment. All patients underwent positron emission tomography/computed tomography (PET/CT) examination, or computed tomography (CT) examination, or magnetic resonance imaging (MRI) examination before starting the initial treatment. Some patients underwent pathological biopsy and immunohistochemistry of extramedullary lesions. Among the 90 patients with extramedullary multiple myeloma, 31 patients received Proteasome inhibitor based (PI-based) regimen, 17 patients received Immunomodulator based (IMiD-based) regimen, 28 patients received PI combined with IMiD regimen, 11 patients were only treated with routine chemotherapy (RC) (including vincristine, adriamycin, cyclophosphamide, doxorubicin liposome, etoposide, etc.) and steroid regimen, and 12 patients received autologous hematopoietic stem cell transplantation (HSCT) .

\section{Clinical Characteristics}

Gender, age, Durie-Salmon stage, ISS stage, hemoglobin, blood calcium, serum creatinine, albumin, lactate dehydrogenase, $\beta 2$-microglobulin, M-protein types, plasma cell percentage and the max of standard uptake value (SUVmax) of PET-CT in extramedullary lesions were analyzed. Some patients were detected by fluorescence in situ hybridization (FISH). The samples were obtained from bone marrow plasma cells sorted by CD138 monoclonal antibody magnetic beads. 1q21 amplification, P53 deletion, IGH translocations, RB1 deletion, the presence of $t(14,16)$ and $t(4,14)$ were detected respectively. Some patients underwent pathological biopsy of extramedullary lesions to detect CD56 and Ki-67.

\section{Efficacy Evaluation}

The efficacy of patients was evaluated according to the criteria of International Myeloma Working Group (Kumar S et al. 2016). The evaluating indicators included the size of plasmacytoma in soft tissue, the proportion of plasmacytoma in bone marrow biopsy, serum and urine $M$ protein, and the $k / \lambda$ ratio, etc. The main efficacy index was the rate of overall remission, which was determined as partial remission or above. 


\section{Survival}

The median follow-up period was 23 months until December 31, 2020. Progression free survival (PFS) was defined as the time from diagnosis to disease progression, recurrence or death. The overall survival (OS) was defined as the time from diagnosis to death or the deadline of follow-up.

\section{Statistical Analysis}

SPSS 23.0 software was used for data analysis. The qualitative variables between patients with single site invasion and multiple sites invasion were compared by Pearson chi-square $(\chi 2)$ test, Continuity correction or Fisher exact test. If the quantitative variables meeted the normal distribution and the variances were homogeneous, the independent-sample t-test was used for analysis. If the distribution was normal but the variances were not homogeneous, the corrected t-test is used. If the normal distribution could not be satisfied, the rank sum test of two independent samples was used for analysis. The data that satisfied normal distribution was described by "mean \pm standard deviation" and the data that did not satisfy normal distribution was described by "median \pm quarter spacing". Graphpad prism 6.0 and the log-rank test was used for survival comparison. A multivariate Cox proportional hazards model was also constructed for survival analysis, and differences were reported as hazard ratios (HRs). Covariates that had a p-value of less than 0.1 in the log-rank test were added to the model. All p-values were 2 -sided and the statistical significance was set at $P<0.05$.

\section{Results}

\section{Clinical Characteristics}

There were 43 (47.8\%) patients with single site invasion, 47 (52.2\%) patients with multiple (two or more) sites invasion. Among 43 patients with single site invasion, 35 patients were extramedullary-bone related, including 13 patients involving sternum and ribs, 11 patients involving vertebral body, 4 patients involving skull, orbital bone or jaw, 3 patients involving ilium, and 4 patients involving limbs. There were also 8 patients extramedullary extraosseous, including 3 patients involving skin soft tissue mass, 2 patients involving pleura, 1 patient involving lymph nodes, 1 patient involving brain, and 1 patient involving breast. Among 47 patients with multiple sites invasion, 21 patients were extramedullary-bone related, with invasion of two or more sites including ribs, vertebral body, limb bones, skull, etc. There were also 26 patients extramedullary extraosseous, with invasion of two or more sites including lymph nodes, abdominal organs, brain, lung, ribs, vertebral body, etc.

Among 43 patients with single site invasion, 18 patients were confirmed by PET/CT examination, 25 patients were confirmed by other imaging examination or bone marrow biopsy. While among 47 patients with multiple sites invasion, 38 patients were confirmed by PET/CT examination, 9 patients were confirmed by other imaging examination or bone marrow biopsy. PET/CT examination showed significant difference in the detection of multiple sites invasion than other imaging examination $(41.9 \%$ vs. $80.9 \%, P=0.000)$.

The Table 1 listed the characteristics of all patients with single site invasion or multiple sites invasion, including age, gender, types of M-protein, hemoglobin, $\beta 2$-microglobulin, albumin, calcium, plasma cell percentage, Durie-Salmon stage, ISS stage, RISS stage, Ki67, FISH results, etc. The results showed that 
patients with multiple sites invasion had higher serum lactate dehydrogenase levels than patients with single site invasion (179.0U/L vs. 154.7U/L, $P=0.016)$. However, no signifificant differences was detected in age, gender, types of $M$ protein, $\beta 2$-microglobulin, albumin, hemoglobin, calcium, creatinine, Durie-Salmon staging, RISS stage, SUVmax, and the FISH results including 1q21 amplification, P53 deletion, IGH translocations, RB1 deletion, the presence of $t(14,16)$ and $t(4,14)$. 
Table 1

Characteristics comparison of newly diagnosed extramedullary multiple myeloma patients between single and multiple sites invasion

\begin{tabular}{|c|c|c|c|}
\hline Characteristics & Single EMM & Multiple EMM & $P$ \\
\hline Age (years) (mean $\pm S D)$ & $58.7 \pm 9.6$ & $57.4 \pm 11.4$ & 0.557 \\
\hline \multicolumn{4}{|l|}{ Gender (n, \%) } \\
\hline Male & $24(55.8 \%)$ & $31(66.0 \%)$ & 0.324 \\
\hline Female & $19(44.2 \%)$ & $16(34.0 \%)$ & \\
\hline \multicolumn{4}{|l|}{ EMM types } \\
\hline EME & $8(18.6 \%)$ & $26(55.3 \%)$ & 0.000 \\
\hline EMB & $35(81.4 \%)$ & $21(44.7 \%)$ & \\
\hline \multicolumn{4}{|l|}{ M-protein types (n, \%) } \\
\hline $\lg A$ & $11(25.6 \%)$ & $16(34.0 \%)$ & 0.221 \\
\hline $\lg G$ & $23(53.5 \%)$ & $18(38.3 \%)$ & \\
\hline $\lg D$ & $0(0 \%)$ & $4(8.5 \%)$ & \\
\hline Light chain & $8(18.6 \%)$ & $7(14.9 \%)$ & \\
\hline Other & $1(2.3 \%)$ & $2(4.3 \%)$ & \\
\hline Hgb (g/L) [Median (IQR)] & $97.0(72.0,127.0)$ & $101.0(82.0,128.0)$ & 0.558 \\
\hline $\begin{array}{l}\text { ß2-microglobulin (g/L) } \\
{[\text { Median (IQR)] }}\end{array}$ & $4.2(2.7,11.3)$ & $5.4(3.0,11.0)$ & 0.368 \\
\hline Albumin (g/L) (mean $\pm S D)$ & $34.1 \pm 8.4$ & $36.1 \pm 6.8$ & 0.216 \\
\hline $\begin{array}{l}\text { Calcium(mmmol/L) } \\
{[\text { Median (IQR)] }}\end{array}$ & $2.2(2.1,2.4)$ & $2.4(2.2,2.5)$ & 0.113 \\
\hline $\begin{array}{l}\text { Creatinine(umol/L) } \\
{[\text { Median (IQR)] }}\end{array}$ & $77.7(54.5,103.8)$ & $91.4(68.4,125.3)$ & 0.074 \\
\hline LDH (U/L) [Median (IQR)] & $154.7(114.7,190.0)$ & $179.0(132.7,299.9)$ & 0.016 \\
\hline $\begin{array}{l}\text { Plasma cell percentage(\%) } \\
\text { [Median (IQR)] }\end{array}$ & $24.0(13.0,56.0)$ & $17.5(7.5,35.4)$ & 0.051 \\
\hline Durie-Salmon stage (n, \%) & & & \\
\hline Stage 1 to 2 & $7(16.3 \%)$ & $10(213 \%)$ & 0.545 \\
\hline Stage 3 & $36(83.7 \%)$ & 37 (78.7\%) & \\
\hline
\end{tabular}




\begin{tabular}{|llll|}
\hline Characteristics & Single EMM & Multiple EMM & $P$ \\
\hline Stage 1 to 2 & $27(62.8 \%)$ & $22(46.8 \%)$ & 0.128 \\
\hline Stage 3 & $16(37.2 \%)$ & $25(53.2 \%)$ & \\
\hline RISS stage (n, \%) & & & 0.206 \\
\hline Stage 1 to 2 & $24(80 \%)$ & $25(67.6 \%)$ & \\
\hline Stage 3 & $6(20 \%)$ & $12(32.4 \%)$ & 0.573 \\
\hline Unknow & 13 & 10 & 0.083 \\
\hline SUVmax [Median (IQR)] & $5.2(3.8,13.5)$ & $7.4(5.0,11.5)$ & 0.650 \\
\hline Ki67 (\%) (mean士SD) & $34.2 \pm 18.0$ & $46.7 \pm 19.7$ & 0.237 \\
\hline CD56 [abnormal /total (\%)] & $7 / 12(58.3 \%)$ & $3 / 8(37.5 \%)$ & 0.180 \\
\hline FISH results & $16(37.2 \%)$ & $25(53.2 \%)$ & 0.648 \\
\hline t(14,16) [abnormal /total (\%)] & $2 / 28(7.1 \%)$ & $0 / 29(0 \%)$ & 0.696 \\
\hline t(4,14) [abnormal /total (\%)] & $5 / 28(17.9 \%)$ & $1 / 29(3.4 \%)$ & 0.301 \\
\hline IGH [abnormal /total (\%)] & $6 / 29(20.7 \%)$ & $5 / 31(16.1 \%)$ & 0.931 \\
\hline P53 [abnormal /total (\%)] & $2 / 30(6.7 \%)$ & $3 / 32(9.4 \%)$ & $6 / 32(18.8 \%)$ \\
\hline RB1 [abnormal /total (\%)] & $9 / 30(30 \%)$ & $11 / 32(34.4 \%)$ & \\
\hline 1q21 [abnormal /total (\%)] & $10 / 30(33.3 \%)$ & & \\
\hline
\end{tabular}

\section{Clinical Efficacy}

Among 43 patients with single site invasion, there were 9 patients with PI-based regimen, 8 patients with IMiDbased regimen, 12 patients with PI combined with IMiD regimen, 2 patients with routine chemotherapy achieving partial remission or above. While among 47 patients with multiple sites invasion, 15 patients with PI-based regimen, 1 patient with IMiD-based regimen, 11 patients with PI combined with IMiD regimen, 3 patients with routine chemotherapy achieved partial remission or above. As a whole, there were 31 patients with single site invasion and 30 patients with multiple sites invasion achieving partial remission or above. No significant difference was showed in the rate of overall remission between patients with single site invasion and multiple sites invasion. Moreover, no matter what chemotherapy regimen the patients received, no significant differences still existed. Among patients with multiple sites invasion, PI-based regimen (84.6\% vs. $33.3 \%, \mathrm{P}=0.035)$ or $\mathrm{PI}$ combined with IMiD regimen (78.9\% vs. $33.3 \%, \mathrm{P}=0.026)$ could achieve superior remission compared with patients with routine chemotherapy. A total of 12 patients underwent hematopoietic stem cell transplantation, including 6 patients with single site invasion and 6 patients multiple sites invasion. All patients achieved partial remission or above. Whether for patients with single site invasion or multiple sites invasion, there were no differences in clinical efficacy between hematopoietic stem cell transplantation and non hematopoietic stem cell transplantation.(Table 2) 
Table 2

Efficacy comparison of of newly diagnosed extramedullary multiple myeloma patients between single and multiple sites invasion

\begin{tabular}{|c|c|c|c|c|c|}
\hline & \multicolumn{2}{|c|}{ Single site invasion $(n, \%)$} & \multicolumn{2}{|c|}{ Multiple sites invasion $(n, \%)$} & \multirow[t]{2}{*}{$p$} \\
\hline & QPR & $\geq \mathrm{PR}$ & QPR & $\geq \mathrm{PR}$ & \\
\hline PI-based & $3(25 \%)$ & $9(75 \%)$ & $4(21.1 \%)$ & $15(78.9 \%)$ & 1.000 \\
\hline IMiD-based & $6(42.9 \%)$ & $8(57.1 \%)$ & $2(66.7 \%)$ & $1(33.3 \%)$ & 0.576 \\
\hline $\mathrm{Pl}+\mathrm{IMiD}$ & $3(20 \%)$ & $12(80 \%)$ & $2(15.4 \%)$ & $11(84.6 \%)$ & 1.000 \\
\hline $\mathrm{RC}$ & $0(0 \%)$ & $2(100 \%)$ & $6(66.7 \%)$ & $3(33.3 \%)$ & 0.182 \\
\hline HSCT & $0(0 \%)$ & $6(100 \%)$ & $0(0 \%)$ & $6(100 \%)$ & - \\
\hline All patients & $12(27.9 \%)$ & $31(72.1 \%)$ & $14(31.8 \%)$ & $30(68.2 \%)$ & 0.690 \\
\hline PI-based vs IMiD-based & $p=0.429$ & & $P=0.169$ & & \\
\hline PI+IMiD vs PI-based & $p=1.000$ & & $P=1.000$ & & \\
\hline PI+IMiD vs IMiD-based & $P=0.245$ & & $P=0.136$ & & \\
\hline PI-based vs RC & $P=1.000$ & & $P=0.035$ & & \\
\hline IMiD-based vs RC & $P=0.500$ & & $P=1.000$ & & \\
\hline $\mathrm{PI}+\mathrm{IMiD}$ vs $\mathrm{RC}$ & $P=1.000$ & & $P=0.026$ & & \\
\hline HSCT VS Non-HSCT & $P=0.163$ & & $P=0.155$ & & \\
\hline
\end{tabular}

\section{Survival of patients with single site invasion}

Among 43 patients with single site invasion, the median PFS was 21 months. The median PFS of of patients with SUVmax $\geq 6.7$ and SUVmax $<6.7$ were 8 months and 27 months respectively, suggesting significantly shorter PFS in patients with SUVmax $\geq 6.7$ ( $\mathrm{HR}=0.280,95 \% \mathrm{Cl}$ : $0.021-0.613, \mathrm{P}=0.024)$. The patient's age, gender, types of M-protein, Durie-Salmon stage, hemoglobin, serum albumin, calcium, creatinine, lactate dehydrogenase, plasma cell percentage, ISS stage and R-ISS stage did not signifificantly affect the PFS of the patients with single site invasion $(P>0.05)$. (Table 3 ) 
Table 3

Survival of newly diagnosed extramedullary multiple myeloma patients with single site invasion

\begin{tabular}{|c|c|c|c|c|c|c|c|}
\hline & & $\begin{array}{l}\text { PFS } \\
\text { (months) }\end{array}$ & HR & $p$ & $\begin{array}{l}\text { OS } \\
\text { (months) }\end{array}$ & HR & $p$ \\
\hline \multirow[t]{2}{*}{ EMM } & EMB & 24 & $\begin{array}{l}0.626(0.194- \\
1.581)\end{array}$ & 0.287 & 42 & $\begin{array}{l}0.453(0.089- \\
1.188)\end{array}$ & 0.100 \\
\hline & EME & 9 & & & 19 & & \\
\hline \multirow[t]{2}{*}{ Age } & $\begin{array}{l}\leq 58 \\
\text { years }\end{array}$ & 21 & $\begin{array}{l}1.198(0.576- \\
2.536)\end{array}$ & 0.625 & NR & $\begin{array}{l}0.582(0.244- \\
1.348)\end{array}$ & 0.213 \\
\hline & $\begin{array}{l}>58 \\
\text { years }\end{array}$ & 21 & & & 32 & & \\
\hline \multirow[t]{2}{*}{ Gender } & Male & 21 & $\begin{array}{l}0.963(0.457- \\
2.021)\end{array}$ & 0.918 & 42 & $\begin{array}{l}0.611(0.250- \\
1.397)\end{array}$ & 0.245 \\
\hline & Female & 23 & & & 30 & & \\
\hline \multirow[t]{2}{*}{$\mathrm{Hgb}(\mathrm{g} / \mathrm{L})$} & $\geq 100$ & 30 & $\begin{array}{l}0.659(0.297- \\
1.347)\end{array}$ & 0.251 & NR & $\begin{array}{l}0.461(0.185- \\
1.042)\end{array}$ & 0.069 \\
\hline & $<100$ & 13 & & & 30 & & \\
\hline \multirow[t]{2}{*}{$\begin{array}{l}\beta 2 \text {-microglobulin } \\
(\mathrm{g} / \mathrm{L})\end{array}$} & $\geq 4.9$ & 10 & $\begin{array}{l}1.665(0.786- \\
4.345)\end{array}$ & 0.175 & 21 & $\begin{array}{l}2.339(1.090- \\
7.326)\end{array}$ & 0.039 \\
\hline & $<4.9$ & 27 & & & NR & & \\
\hline \multirow[t]{2}{*}{ Albumin (g/L) } & $\geq 35.7$ & 21 & $\begin{array}{l}1.252(0.604- \\
2.662)\end{array}$ & 0.541 & 42 & $\begin{array}{l}0.801(0.335- \\
1.855)\end{array}$ & 0.600 \\
\hline & $<35.7$ & 21 & & & 32 & & \\
\hline \multirow[t]{2}{*}{ Calcium(mmmol/L) } & $\geq 2.3$ & 16 & $\begin{array}{l}1.379(0.667- \\
2.993)\end{array}$ & 0.380 & 32 & $\begin{array}{l}1.730(0.754- \\
4.360)\end{array}$ & 0.194 \\
\hline & $<2.3$ & 30 & & & NR & & \\
\hline \multirow[t]{2}{*}{ Creatinine(umol/L) } & $\geq 84.2$ & 18 & $\begin{array}{l}1.139(0.544- \\
2.425)\end{array}$ & 0.724 & 30 & $\begin{array}{l}1.297(0.554- \\
3.147)\end{array}$ & 0.541 \\
\hline & $<84.2$ & 27 & & & 42 & & \\
\hline \multirow[t]{2}{*}{ LDH (U/L) } & $\geq 165.7$ & 16 & $\begin{array}{l}1.548(0.733- \\
3.719)\end{array}$ & 0.241 & 19 & $\begin{array}{l}1.766(0.739- \\
5.218)\end{array}$ & 0.188 \\
\hline & $<165.7$ & 23 & & & 46 & & \\
\hline \multirow[t]{2}{*}{$\begin{array}{l}\text { Percentage of } \\
\text { Plasma cell (\%) }\end{array}$} & $\geq 20$ & 18 & $\begin{array}{l}1.449(0.706- \\
3.112)\end{array}$ & 0.314 & 32 & $\begin{array}{l}2.289(0.991- \\
5.507)\end{array}$ & 0.059 \\
\hline & $<20$ & 30 & & & NR & & \\
\hline Durie-Salmon stage & $1-2$ & 30 & $\begin{array}{l}0.920(0.358- \\
2.354)\end{array}$ & 0.862 & 30 & $\begin{array}{l}1.166(0.377- \\
3.716)\end{array}$ & 0.778 \\
\hline
\end{tabular}




\begin{tabular}{|c|c|c|c|c|c|c|c|}
\hline & & $\begin{array}{l}\text { PFS } \\
\text { (months) }\end{array}$ & HR & $p$ & $\begin{array}{l}\text { OS } \\
\text { (months) }\end{array}$ & HR & $p$ \\
\hline & 3 & 21 & & & 42 & & \\
\hline \multirow[t]{2}{*}{ ISS stage } & 1-2 & 26 & $\begin{array}{l}0.646(0.257- \\
1.378)\end{array}$ & 0.244 & NR & $\begin{array}{l}0.437(0.141- \\
0.942)\end{array}$ & 0.044 \\
\hline & 3 & 10 & & & 21 & & \\
\hline \multirow[t]{2}{*}{ RISS } & $1-2$ & 23 & $\begin{array}{l}0.467(0.081- \\
1.417)\end{array}$ & 0.153 & 46 & $\begin{array}{l}0.381(0.051- \\
1.109)\end{array}$ & 0.081 \\
\hline & 3 & 13 & & & 22 & & \\
\hline \multirow[t]{2}{*}{ SUVmax } & $\geq 6.7$ & 8 & $\begin{array}{l}0.280(0.021- \\
0.613)\end{array}$ & 0.024 & 16 & $\begin{array}{l}0.175(0.013- \\
0.519)\end{array}$ & 0.014 \\
\hline & $<6.7$ & 27 & & & NR & & \\
\hline
\end{tabular}

Among 43 patients with single site invasion, the median OS was 42 months. The median OS of patients with $\beta 2$-microglobulin $\geq 4.9 \mathrm{~g} / \mathrm{L}$ was 21 months, while with $\beta 2$-microglobulin $<4.9 \mathrm{~g} / \mathrm{L}$ was not reached, suggesting significantly shorter OS in patients with $\beta 2$-microglobulin $\geq 4.9 \mathrm{~g} / \mathrm{L}(\mathrm{HR}=2.339,95 \% \mathrm{Cl}$ : 1.090-7.326, $\mathrm{P}=0.039)$. The median OS of patients with ISS stage 1-2 was not reached, and with stage 3 was 21 months, suggesting significantly longer OS in patients with ISS stage $1-2(\mathrm{HR}=0.437,95 \% \mathrm{Cl}: 0.141-0.942, \mathrm{P}=0.044)$. The median OS of patients with SUVmax $\geq 6.7$ was 16 months, with SUVmax $<6.7$ was not reached, suggesting significantly longer OS in patients with SUVmax $<6.7(\mathrm{HR}=0.175,95 \% \mathrm{Cl}: 0.013-0.519, \mathrm{P}=0.014)$. In addition, $\mathrm{PI}$ combined with IMiD regimen could improve PFS ( $\mathrm{HR}=0.313,95 \% \mathrm{Cl}$ : $0.116-0.795, \mathrm{P}=0.018)$ and $\mathrm{OS}(\mathrm{HR}=0.146,95 \% \mathrm{Cl}$ : 0.057-0.507, $\mathrm{P}=0.002$ ) of patients with single site invasion compared with IMiD-based regimen. HSCT was also associated with the improved OS ( $\mathrm{HR}=0.000,95 \% \mathrm{Cl}$ : 0.091-0.860, $\mathrm{P}=0.030)$. Age, gender, M-protein types, Durie-Salmon stage, hemoglobin, serum albumin, calcium, creatinine, lactate dehydrogenase, plasma cell percentage and RISS stage did not signifificantly affect the OS of patients with single site invasion $(P>0.05)$. (Table 3, Kaplan-Meier survival curves were showed in Figure 1)

\section{Survival of patients with multiple sites invasion}

Among 47 patients with multiple sites invasion, the median PFS was 18 months. The median PFS of EME patients was 18 months, of EMB patients was 47 months, suggesting significantly shorter PFS in EME patients ( $\mathrm{HR}=2.108,95 \% \mathrm{Cl}: 1.053-4.765, \mathrm{P}=0.048)$. The median PFS of male patients was 33 months, significantly longer than female patients $(\mathrm{HR}=0.386,95 \% \mathrm{Cl}: 0.113-0.667, \mathrm{P}=0.007)$. The median PFS of patients with ISS stage 3 and RISS stage 3 were 11 and 9 months respectively, which decreased significantly compared with ISS stage $1-2(\mathrm{HR}=0.443,95 \% \mathrm{Cl}: 0.160-0.804, \mathrm{P}=0.020)$ and RISS stage $1-2(\mathrm{HR}=0.283,95 \% \mathrm{Cl}$ : $0.035-0.397, P=0.001$ ). The median PFS of of patients with $\beta 2$-microglobulin $\geq 4.9 \mathrm{~g} / \mathrm{L}$ and $\beta 2$ microglobulin $<4.9 \mathrm{~g} / \mathrm{L}$ were 13 and 33 months respectively, suggesting shorter PFS in patients with $\beta 2$ microglobulin $\geq 4.9 \mathrm{~g} / \mathrm{L}(\mathrm{HR}=2.613,95 \% \mathrm{Cl}: 1.479-7.140, \mathrm{P}=0.006)$. Among patients with $\mathrm{LDH} \geq 165.7 \mathrm{U} / \mathrm{L}$, the median PFS was 16 months, 
significantly shorter than $\mathrm{LDH}<165.7 \mathrm{U} / \mathrm{L}(\mathrm{HR}=2.487,95 \% \mathrm{Cl}$ : 1.110-5.131, $\mathrm{P}=0.033)$. In addition, HSCT was also associated with improved PFS ( $H R=0.000,95 \% \mathrm{Cl}$ : 0.102-0.734, $\mathrm{P}=0.013$ ). The patient's age, types of $\mathrm{M}$ protein, albumin, hemoglobin, Durie-Salmon stage, calcium, creatinine and SUVmax did not signifificantly affect the PFS of the patients with multiple sites invasion $(P>0.05)$ (Table 4, Kaplan-Meier survival curves were showed in Figure 2). 
Table 4

Survival of newly diagnosed extramedullary multiple myeloma patients with multiple sites invasion

\begin{tabular}{|c|c|c|c|c|c|c|c|}
\hline & & $\begin{array}{l}\text { PFS } \\
\text { (months) }\end{array}$ & $\mathrm{HR}(95 \% \mathrm{Cl})$ & $p$ & $\begin{array}{l}\text { OS } \\
\text { (months) }\end{array}$ & $\mathrm{HR}(95 \% \mathrm{Cl})$ & $p$ \\
\hline \multirow[t]{2}{*}{ EMM } & EME & 18 & $\begin{array}{l}2.108(1.053- \\
4.765)\end{array}$ & 0.048 & 30 & $\begin{array}{l}1.890(0.793- \\
4.575)\end{array}$ & 0.159 \\
\hline & EMB & 47 & & & 47 & & \\
\hline \multirow[t]{2}{*}{ Age } & $\begin{array}{l}\leq 58 \\
\text { years }\end{array}$ & 26 & $\begin{array}{l}1.096(0.519- \\
2.357)\end{array}$ & 0.803 & 36 & $\begin{array}{l}1.514(0.635- \\
3.705)\end{array}$ & 0.347 \\
\hline & $\begin{array}{l}>58 \\
\text { years }\end{array}$ & 18 & & & 47 & & \\
\hline \multirow[t]{2}{*}{ Gender } & Male & 33 & $\begin{array}{l}0.386(0.113- \\
0.667)\end{array}$ & 0.007 & 47 & $\begin{array}{l}0.398(0.114- \\
0.873)\end{array}$ & 0.030 \\
\hline & Female & 13 & & & 25 & & \\
\hline \multirow[t]{2}{*}{ M-protein } & $\lg D$ & 7 & $\begin{array}{l}1.883(0.511- \\
11.59)\end{array}$ & 0.279 & 29 & $\begin{array}{l}1.985(0.514- \\
12.62)\end{array}$ & 0.258 \\
\hline & $\begin{array}{l}\text { non- } \\
\text { lgD }\end{array}$ & 18 & & & 36 & & \\
\hline \multirow[t]{2}{*}{$\mathrm{Hb}(\mathrm{g} / \mathrm{L})$} & $\geq 100$ & 33 & $\begin{array}{l}0.720(0.326- \\
1.479)\end{array}$ & 0.369 & NR & $\begin{array}{l}0.535(0.217- \\
1.267)\end{array}$ & 0.157 \\
\hline & $<100$ & 18 & & & 28 & & \\
\hline \multirow[t]{2}{*}{$\begin{array}{l}\text { B2-microglobulin } \\
(\mathrm{g} / \mathrm{L})\end{array}$} & $\geq 4.9$ & 13 & $\begin{array}{l}2.613(1.479- \\
7.140)\end{array}$ & 0.006 & 20 & $\begin{array}{l}4.920(2.207- \\
13.45)\end{array}$ & 0.000 \\
\hline & $<4.9$ & 33 & & & NR & & \\
\hline \multirow[t]{2}{*}{ Albumin $(\mathrm{g} / \mathrm{L})$} & $\geq 35.7$ & 18 & $\begin{array}{l}1.233(0.559- \\
2.722)\end{array}$ & 0.604 & 36 & $\begin{array}{l}1.382(0.575- \\
3.321)\end{array}$ & 0.474 \\
\hline & $<35.7$ & 18 & & & 47 & & \\
\hline \multirow[t]{2}{*}{ Calcium(mmmol/L) } & $\geq 2.3$ & 13 & $\begin{array}{l}1.725(0.847- \\
3.843)\end{array}$ & 0.151 & 36 & $\begin{array}{l}1.157(0.470- \\
2.848)\end{array}$ & 0.754 \\
\hline & $<2.3$ & 26 & & & 47 & & \\
\hline \multirow[t]{2}{*}{ Creatinine(umol/L) } & $\geq 84.2$ & 16 & $\begin{array}{l}1.447(0.703- \\
3.177)\end{array}$ & 0.320 & 30 & $\begin{array}{l}1.348(0.561- \\
3.246)\end{array}$ & 0.507 \\
\hline & $<84.2$ & 26 & & & 47 & & \\
\hline \multirow[t]{2}{*}{$\mathrm{LDH}(\mathrm{U} / \mathrm{L})$} & $\geq 165.7$ & 16 & $\begin{array}{l}2.487 \\
(1.110- \\
5.131)\end{array}$ & 0.033 & 25 & $\begin{array}{l}3.005(1.072- \\
6.298)\end{array}$ & 0.037 \\
\hline & $<165.7$ & NR & & & NR & & \\
\hline
\end{tabular}




\begin{tabular}{|c|c|c|c|c|c|c|c|}
\hline & & $\begin{array}{l}\text { PFS } \\
\text { (months) }\end{array}$ & $\mathrm{HR}(95 \% \mathrm{Cl})$ & $p$ & $\begin{array}{l}\text { OS } \\
\text { (months) }\end{array}$ & $\mathrm{HR}(95 \% \mathrm{Cl})$ & $p$ \\
\hline \multirow[t]{2}{*}{$\begin{array}{l}\text { Percentage of } \\
\text { Plasma cell (\%) }\end{array}$} & $\geq 20$ & 18 & $\begin{array}{l}1.543 \\
(0.731- \\
3.787)\end{array}$ & 0.248 & 22 & $\begin{array}{l}2.298(1.071- \\
7.213)\end{array}$ & 0.045 \\
\hline & $<20$ & 26 & & & 47 & & \\
\hline \multirow[t]{2}{*}{ Durie-Salmon stage } & $1-2$ & 26 & $\begin{array}{l}0.938 \\
(0.383- \\
2.279)\end{array}$ & 0.886 & NR & $\begin{array}{l}0.294(0.153- \\
1.090)\end{array}$ & 0.077 \\
\hline & 3 & 18 & & & 36 & & \\
\hline \multirow[t]{2}{*}{ ISS stage } & $1-2$ & 33 & $\begin{array}{l}0.443 \\
(0.160- \\
0.804)\end{array}$ & 0.020 & 47 & $\begin{array}{l}0.282(0.092- \\
0.582)\end{array}$ & 0.003 \\
\hline & 3 & 11 & & & 18 & & \\
\hline \multirow[t]{2}{*}{ R-ISS } & $1-2$ & 30 & $\begin{array}{l}0.283 \\
(0.035- \\
0.397)\end{array}$ & 0.001 & 47 & $\begin{array}{l}0.150 \\
(0.007- \\
0.123)\end{array}$ & 0.000 \\
\hline & 3 & 9 & & & 10 & & \\
\hline \multirow[t]{2}{*}{ SUVmax } & $\geq 6.7$ & 18 & $\begin{array}{l}0.749 \\
(0.278- \\
1.874)\end{array}$ & 0.522 & 43 & $\begin{array}{l}0.782(0.269- \\
2.223)\end{array}$ & 0.639 \\
\hline & $<6.7$ & 15 & & & 28 & & \\
\hline
\end{tabular}

Table 5

The survival of patients with different treatment regimens

\begin{tabular}{|lllll|}
\hline \multicolumn{4}{l}{ Single site invasion } & \multicolumn{2}{l|}{ Multiple sites invasion } \\
\hline Treatment schemes & PFS & OS & PFS & OS \\
(months) & (months) & (months) & (months) \\
\hline PI-based & 24 & 32 & 18 & 47 \\
\hline IMiD-based & 11 & 19 & 21.5 & 34 \\
\hline PI+IMiD & NR & NR & 18 & 36 \\
\hline RC & 12.5 & 29 & 11 & 20 \\
\hline HSCT & 30 & NR & NR & NR \\
\hline Non-HSCT & 18 & 30 & 16 & 30 \\
\hline $\begin{array}{l}P / \text { proteasome inhibitors, IMiD immunomodulators, HSCT autologous hematopoietic stem cell } \\
\text { transplantation, NR not reached, } R C \text { routine chemotherapy }\end{array}$ & & \\
\hline
\end{tabular}


Table 6

The survival comparison of different treatment regimens in newly diagnosed extramedullary multiple myeloma patients between single and multiple sites invasion

\begin{tabular}{|c|c|c|c|c|c|c|c|c|}
\hline & \multicolumn{4}{|c|}{ Single site invasion } & \multicolumn{4}{|c|}{ Multiple sites invasion } \\
\hline & $\mathrm{HR}^{\mathrm{a}}(95 \% \mathrm{Cl})$ & $p^{a}$ & $\begin{array}{l}\mathrm{HR}^{\mathrm{b}} \\
(95 \% \mathrm{Cl})\end{array}$ & $p^{b}$ & $\begin{array}{l}\mathrm{HR}^{\mathrm{a}} \\
(95 \% \mathrm{Cl})\end{array}$ & $p^{a}$ & $\begin{array}{l}\mathrm{HR}^{\mathrm{b}} \\
(95 \% \mathrm{Cl})\end{array}$ & $p^{b}$ \\
\hline $\begin{array}{l}\text { PI-based vs } \\
\text { IMiD-based }\end{array}$ & $\begin{array}{l}0.732 \\
(0.305- \\
1.686)\end{array}$ & 0.461 & $\begin{array}{l}0.510 \\
(0.189- \\
1.266)\end{array}$ & 0.158 & $\begin{array}{l}0.672 \\
(0.108- \\
3.572)\end{array}$ & 0.599 & $\begin{array}{l}0.490 \\
(0.053- \\
2.812)\end{array}$ & 0.355 \\
\hline $\begin{array}{l}\text { Pl+IMiD vs } \\
\text { Pl-based }\end{array}$ & $\begin{array}{l}0.442(0.149- \\
1.228)\end{array}$ & 0.123 & $\begin{array}{l}0.264 \\
(0.071- \\
1.155)\end{array}$ & 0.079 & $\begin{array}{l}1.019 \\
(0.388- \\
2.686)\end{array}$ & 0.968 & $\begin{array}{l}1.114 \\
(0.351- \\
3.566)\end{array}$ & 0.852 \\
\hline $\begin{array}{l}\text { PI+IMiD vs } \\
\text { IMiD-based }\end{array}$ & $\begin{array}{l}0.313 \\
(0.116- \\
0.795)\end{array}$ & 0.018 & $\begin{array}{l}0.146 \\
(0.057- \\
0.507)\end{array}$ & 0.002 & $\begin{array}{l}0.616 \\
(0.085- \\
3.305)\end{array}$ & 0.519 & $\begin{array}{l}0.798 \\
(0.137- \\
4.358)\end{array}$ & 0.779 \\
\hline $\begin{array}{l}\text { Pl-based vs } \\
\text { RC }\end{array}$ & $\begin{array}{l}0.384 \\
(0.025- \\
1.990)\end{array}$ & 0.191 & $\begin{array}{l}0.581 \\
(0.078- \\
3.351)\end{array}$ & 0.492 & $\begin{array}{l}0.440 \\
(0.127- \\
1.043)\end{array}$ & 0.067 & $\begin{array}{l}0.441 \\
(0.112- \\
1.284)\end{array}$ & 0.124 \\
\hline $\begin{array}{l}\text { IMiD-based } \\
\text { vs RC }\end{array}$ & $\begin{array}{l}0.798 \\
(0.151- \\
3.907)\end{array}$ & 0.759 & $\begin{array}{l}0.861 \\
(0.171- \\
4.092)\end{array}$ & 0.835 & $\begin{array}{l}0.687 \\
(0.169- \\
2.784)\end{array}$ & 0.615 & $\begin{array}{l}0.671 \\
(0.155- \\
2.942)\end{array}$ & 0.612 \\
\hline $\begin{array}{l}\mathrm{Pl}+\mathrm{IMiD} \text { vs } \\
\mathrm{RC}\end{array}$ & $\begin{array}{l}0.236 \\
(0.005- \\
1.039)\end{array}$ & 0.057 & $\begin{array}{l}0.203 \\
(0.006- \\
1.093)\end{array}$ & 0.067 & $\begin{array}{l}0.552 \\
(0.165- \\
1.319)\end{array}$ & 0.196 & $\begin{array}{l}0.438 \\
(0.118- \\
1.415)\end{array}$ & 0.159 \\
\hline $\begin{array}{l}\text { HSCT VS } \\
\text { Non-HSCT }\end{array}$ & $\begin{array}{l}0.504 \\
(0.219- \\
1.435)\end{array}$ & 0.240 & $\begin{array}{l}0.000 \\
(0.091- \\
0.860)\end{array}$ & 0.030 & $\begin{array}{l}0.000 \\
(0.102- \\
0.734)\end{array}$ & 0.013 & $\begin{array}{l}0.000 \\
(0.092- \\
0.957)\end{array}$ & 0.043 \\
\hline \multicolumn{9}{|c|}{$\begin{array}{l}\text { P/ proteasome inhibitors, IMiD immunomodulators, } H S C T \text { autologous hematopoietic stem cell } \\
\text { transplantation, } N R \text { not reached, } R C \text { routine chemotherapy, }\end{array}$} \\
\hline
\end{tabular}

Among 47 patients with multiple sites invasion, the median OS was 36 months. The median OS of male patients was 47 months, significantly longer than female patients ( $\mathrm{HR}=0.398,95 \% \mathrm{Cl}$ : $0.114-0.873, \mathrm{P}=0.030)$. The median OS of patients with $\beta 2$-microglobulin $\geq 4.9 \mathrm{~g} / \mathrm{L}$ was 20 months, with $\beta 2$-microglobulin $<4.9 \mathrm{~g} / \mathrm{L}$ was not reached, suggesting significantly shorter $O S$ in patients with $\beta 2$-microglobulin $\geq 4.9 \mathrm{~g} / \mathrm{L}(\mathrm{HR}=4.920,95 \% \mathrm{Cl}$ : 2.207-13.45, $\mathrm{P}=0.000$ ). Among patients with $\mathrm{LDH} \geq 165.7 \mathrm{U} / \mathrm{L}$, the median PFS was 25 months, significantly shorter than $\mathrm{LDH}<165.7 \mathrm{U} / \mathrm{L}(\mathrm{HR}=$

3.005, 95\% Cl: $1.072-6.298, \mathrm{P}=0.037)$. The median OS of patients with ISS stage $1-2$ and RISS stage $1-2$ were all 47 months, significantly longer than the OS of ISS stage $3(\mathrm{HR}=0.282,95 \% \mathrm{Cl}: 0.092-0.582, \mathrm{P}=0.003)$ and RISS stage $3(\mathrm{HR}=0.150,95 \% \mathrm{Cl}: 0.007-0.123, \mathrm{P}=0.000)$. The median OS of patients with plasma cell percentage $\geq 20 \%$ and $<20 \%$ were 22 and 47 months respectively, suggesting significantly shorter OS in patients with plasma cell percentage $\geq 20 \%(H R=2.298,95 \% \mathrm{Cl}: 1.071-7.213, \mathrm{P}=0.045)$. HSCT was also 
associated with improved OS ( $\mathrm{HR}=0.000,95 \% \mathrm{Cl}: 0.092-0.957, \mathrm{P}=0.043)$. Age, types of M protein, Durie-Salmon stage, hemoglobin, albumin, calcium, creatinine, and SUVmax did not signifificantly affect the OS of the patients with multiple sites invasion ( $P>0.05$ ). (Table 4, Kaplan-Meier survival curves were showed in Figure 3 ).

Among newly diagnosed extramedullary multiple myeloma patients with single site invasion, the results of multivariate survival analysis showed that proteasome inhibitors combined with immunomodulators regimen could significantly improve the PFS ( $\mathrm{HR}=0.080,95 \% \mathrm{Cl}$ : $0.007-0.855, \mathrm{P}=0.037)$. Among patients with multiple sites invasion, the results of multivariate survival analysis showed that RISS 3 was associated with poor PFS $(\mathrm{HR}=4.081,95 \% \mathrm{Cl}: 1.533-10.865, \mathrm{P}=0.005)$ and $\mathrm{OS}(\mathrm{HR}=13.295,95 \% \mathrm{Cl}: 3.219-54.907, \mathrm{P}=0.000)$.

\section{Discussion}

Extramedullary multiple myeloma is a rare multiple myeloma in which myeloma cells are independent of the micro-environment of bone marrow, infiltrate surrounding tissues and/or circulate freely in the blood, involving lymph nodes, skin and soft tissues, central nervous system, thoracic, abdominal organs, and any other anatomical sites. Extramedullary multiple myeloma can be present at initial diagnosis with an estimated incidence of $7 \%$ or at relapse with an estimated incidence of $6 \%$ (Varettoni $\mathrm{M}$ et al. 2010), with the overall incidence of $18.2 \%$ (Gagelmann N et al 2018). Due to the development of modern imaging technology, especially the widespread application of enhanced CT, MRI and PET-CT, the detection rate of EMM has increased. Among patients with multiple myeloma, the diagnosis of EMM is typically made by the presence of pathologic soft tissue masses based on biopsy and imaging technology, including PET/CT, CT, MRI or ultrasound (Touzeau C and Moreau P 2016). In our study, there were 56 (62.2\%) patients confirmed by PET/CT examination, including 18 patients with single site invasion and 38 patients with multiple sites invasion. $\mathrm{PET} / \mathrm{CT}$ examination showed signifificant difference in the detection of multiple sites invasion than other imaging examination. PET/CT has become an important imaging technique for EMM patients.

The patients possibly had single site invasion, possibly had two or more sites invasion. In our study, there were $47.8 \%$ patients with single site invasion, $52.2 \%$ patients with multiple sites invasion. The results of our study showed that patients with multiple sites invasion had significantly higher proportion (55.3\%) of EME than patients with single site invasion. However, in the study carried by Batsukh $\mathrm{K}$ et al.(2017), multiple sites invasion was more common in parabosseous patients, with a proportion of $67 \%$. He JS et al. analyzed the clinical characteristics of $80 \mathrm{EMM}$ patients and 277 patients without EMM, they showed elevated serum lactate dehydrogenase in EMM patients (32.5\% vs. $16.2 \%, P=0.001)$ (He JS et al. 2021). In another study, EME patients had significantly higher lactate dehydrogenase than EMB patients ( $256 \mathrm{U} / \mathrm{L}$ vs $184 \mathrm{U} / \mathrm{L}, \mathrm{P}=0.003)$ (Wang $\mathrm{J}$ et al. 2020). In our study, we found the significant difference in lactate dehydrogenase between patients with single site invasion and multiple sites invasion, suggesting possibly high tumor load in patients with multiple sites invasion. Although higher $\beta 2$-microglobulin level in extramedullary disease, more prevalent $\mathrm{t}(4 ; 14)$ in extramedullary plasma cells, and higher 1q21 amplification rate in EME patients were found in other studies (Wang J et al. 2020; Tian C et al. 2018; Besse L et al. 2016), we did not find these differences in patients with single site invasion and multiple sites invasion. Moreover, no signifificant differences were also detected in age, gender, types of M protein, albumin, hemoglobin, calcium, creatinine, Durie-Salmon stage, RISS stage, etc. 
The emergence of new drugs including PI, IMiD and monoclonal antibody has improved the survival of patients with multiple myeloma and brought hope to clinical treatment. However, there is still no consensus on the best treatment option for patients with extramedullary multiple myeloma. Among 38 patients with multiple myeloma reported by Rosinol et al, 11 patients with extramedullary multiple myeloma had no response to thalidomide ( Rosiñol L et al. 2004). Laura R et al.(2006) reported that 4 patients with extramedullary multiple myeloma who failed to thalidomide treatment were treated with bortezomib, and 3 patients with large soft tissue plasma cell tumors disappeared. Among our patients, 61 patients achieved partial remission or above, and the total remission rate of new drugs as induction therapy was $67.8 \%$. We conducted the efficacy comparison of the same regimen between patients with single site invasion and multiple sites invasion, and the results showed no significant differences. We also compared the efficacy of different regimens in patients with single site invasion, and in patients with multiple sites invasion. The results suggested the superiority of $\mathrm{PI}$ or $\mathrm{PI}$ combined with IMiD in overall remission rate compared with routine chemotherapy. A total of 12 patients with hematopoietic stem cell transplantation achieved partial remission or above, including 6 patients with single site invasion and 6 patients with multiple sites invasion. But no significant differences were found between patients with HSCT and Non-HSCT possibly as a result of fewer transplant patients.

Among 43 patients with single site invasion, the median PFS was 21 months. The univariate analysis of survival showed that patients with SUVmax $\geq 6.7$ had significantly shorter PFS. In these patients with single site invasion, the median OS was 42 months. Patients with $\beta 2$-microglobulin $\geq 4.9 \mathrm{~g} / \mathrm{L}$, SUVmax $\geq 6.7$, ISS stage 3 had poor OS. Among 47 patients with multiple sites invasion, the median PFS was 18 months. The univariate analysis of survival showed that patients with extramedullary extraosseous, female gender, $\beta 2$ microglobulin $\geq 4.9 \mathrm{~g} / \mathrm{L}, \mathrm{LDH} \geq 165.7 \mathrm{U} / \mathrm{L}$, ISS stage 3 and RISS stage 3 had significantly shorter PFS. In these patients with multiple sites invasion, the median OS 36 months. Patients with female gender, $\beta 2-$ microglobulin $\geq 4.9 \mathrm{~g} / \mathrm{L}, \mathrm{LDH} \geq 165.7 \mathrm{U} / \mathrm{L}$, plasma cell percentage $\geq 20 \%$, ISS stage 3 and RISS stage 3 also had poor OS. The study of Zhang $Y$ et al.(2017) reported that serum lactate dehydrogenase and $\beta 2$-microglobulin were independent risk factors for OS of newly diagnosed multiple myeloma. In another study, multiple myeloma patients with high LDH levels had higher serum $\beta 2$-microglobulin and a higher percentage of extramedullary relapse compared with patients with normal LDH levels (Liu Y et al. 2020). One retrospective study carried in patients with multiple myeloma, the survival rate was lower in patients with higher ISS stage (Andriandi et al. 2019). Surprisingly, we proved the effect of $\beta 2$-microglobulin, LDH and ISS stage on the PFS or OS in patients with single site invasion or multiple sites invasion. Especially, the median HR value was more larger or smaller in patients with multiple sites invasion, suggesting this effect is more obvious in patients with multiple sites invasion. In addition, we found the association of female gender with the poor PFS and OS in extramedullary multiple myeloma patients with multiple sites invasion. Guillermo CL et al. reported that male sex was one of predictive factors for early mortality of multiple myeloma patients (Guillermo CL et al. 2007). Other studies also confirmed the association of female gender with improved survival (Jayakrishnan TT et al. 2021; Ikeda T et al. 2019). But in another study, female gender was confirmed to be associated with inferior overall survival (44.8 months vs. 49.9 months, $P=0.020$ ) (Boyd KD et al. 2011).

We also analyzed the survival of patients with different treatment regimens. We found that PI combined with IMiD regimen was superior to IMiD in improving PFS and OS in patients with single site invasion. Cox multivariate survival analysis aslo showed the improved PFS in EMM patients with single site invasion. A

Page 16/23 
phase 2 study reported that the ORR was 94\% in transplant-ineligible multiple myeloma patients after 4 cycles of lenalidomide, bortezomib and dexamethasone therapy. After the follow-up time, the median PFS was 35.1 months, and the median OS was not reached ( O'Donnell EK et al. 2018). Kumar SK et al. proved that bortezomib, lenalidomide, and dexamethasone triplet regimen remained the standard of care for induction therapy for patients with standard-risk and intermediate-risk newly diagnosed multiple myeloma by comparison with another triplet regimen (carfilzomib, lenalidomide and dexamethasone) (Kumar SK et al. 2020). Referring to these results and the results in our study, the regimen of proteasome inhibitors combined with immunomodulators regimen could improve the survival of extramedullary multiple myeloma patients with single site invasion. In addition to these findings, we also found the obvious advantage of autologous hematopoietic stem cell transplantation in improving the survival of extramedullary multiple myeloma patients. In the study carried by Czyż J. et al., autologous stem cell transplantation was an important factor in improving survival ( $\mathrm{HR}=3.23 ; 95 \% \mathrm{Cl}$ : 1.52-6.84) in patients with multiple myeloma with 17p deletion (Czyż $\mathrm{J}$ et al. 2020). For newly diagnosed multiple myeloma paitients, autologous HSCT could improve the PFS (Cavo M et al. 2020). Moreover, a study from the chronic malignancies working party of the european society for blood and marrow transplantation showed that tandem autologous stem cell transplantation could significantly improve outcomes in newly diagnosed multiple myeloma patients with extramedullary disease and high-risk cytogenetics (Gagelmann N et al. 2019). However, the multivariate survival analysis only found the association of RISS stage 3 with the poor survival of newly diagnosed extramedullary multiple myeloma patients.

We have achieved some promising results in newly diagnosed extramedullary multiple myeloma patients with single site invasion, such as the improved survival of proteasome inhibitors combined with immunomodulators regimen. Moreover, in patients with multiple sites invasion, the association of RISS stage 3 with poor survival was also comfirmed. Our research also had some limitations. First, the data was obtained from a retrospective analysis. Secondly, the numbers of patients with some characteristics were very small, such as the number of patients with stem cell transplantation, the number of patients with routine chemotherapy, possibly affected the significance of the results. Therefore, we propose a prospective, large sample clinical study to explore the effect of new drugs and stem cell transplantation on survival of patients with newly diagnosed extramedullary multiple myeloma, especially patients at RISS stage 3 .

\section{Declarations}

\section{Acknowledgements}

We acknowledged all doctors participated in the diagnosis and treatment of extramedullary multiple myeloma patients in Zhongnan Hospital of Wuhan University.

\section{Author contributions}

YZ participated in data arrangement, statistical analysis, and wrote the manuscript. FZ designed the project, provided professional guidance, and revised the manuscript.

\section{Funding}


This study has been partially supported by Innovation Fund of WNLO (2018WNLOKF023).

\section{Data availability}

The data and material are available from the corresponding author upon reasonable request.

\section{Compliance with ethical standards}

\section{Conflict of interest}

All authors declare that they have no conficts of interest related to this manuscript. All authors have neither relevant commercial interests nor fnancial or material support to disclose. All authors have contributed signifcantly, and all authors are in agreement with the content of the manuscript.

\section{Ethical approval}

This is a retrospective study. All private information about the included patients were erased and the requirement for written informed consent is waived by the the Institutional Review Board of the Wuhan University, China.

\section{References}

Andriandi et al (2019) Survival rate of multiple myeloma patients in Indonesia: A retrospective study in multiple myeloma at a single institution. Ann Med Surg (Lond) 41:11-15. https://doi:

10.1016/j.amsu.2019.03.011

Avivi I et al (2019) Hematogenous extramedullary relapse in multiple myeloma - a multicenter retrospective study in 127 patients. Am J Hematol 94(10):1132-1140. https://doi: 10.1002/ajh.255

Batsukh K et al (2017) Distinct Clinical Outcomes between Paramedullary and Extramedullary Lesions in Newly Diagnosed Multiple Myeloma. Immune Netw 17(4):250-260. https://doi: 10.4110/in.2017.17.4.250

Besse $L$ et al (2016) Cytogenetics in multiple myeloma patients progressing into extramedullary disease. Eur $J$ Haematol 97(1):93-100. https:// doi: 10.1111/ejh.12688.

Bhutani M et al (2020) Extramedullary multiple myeloma. Leukemia 34(1):1-20. https://doi: 10.1038/s41375019-0660-0

Boyd KD et al (2011) Gender disparities in the tumor genetics and clinical outcome of multiple myeloma. Cancer Epidemiol Biomarkers Prev 20(8):1703-7. https://doi: 10.1158/1055-9965

Cavo M et al (2020) Autologous haematopoietic stem-cell transplantation versus bortezomib-melphalanprednisone, with or without bortezomib-lenalidomide-dexamethasone consolidation therapy, and lenalidomide maintenance for newly diagnosed multiple myeloma (EMN02/H095): a multicentre, randomised, open-label, phase 3 study. Lancet Haematol 7(6):e456-e468. https://doi: 10.1016/S2352-3026(20)30099-5 
Czyż J et al (2020) Autologous stem cell transplantation in the treatment of multiple myeloma with 17p deletion. Pol Arch Intern Med 130(2):106-111. https://doi: 10.20452/pamw.15139

Impact of extramedullary disease in patients with newly diagnosed multiple myeloma undergoing autologous stem cell transplantation: a study from the Chronic Malignancies Working Party of the EBMT. Haematologica 103(5):890-897. https://doi: 10.3324/haematol

Gagelmann N et al (2019) Tandem Autologous Stem Cell Transplantation Improves Outcomes in Newly Diagnosed Multiple Myeloma with Extramedullary Disease and High-Risk Cytogenetics: A Study from the Chronic Malignancies Working Party of the European Society for Blood and Marrow Transplantation. Biol Blood Marrow Transplant 25(11):2134-2142. https://doi: 10.1016/j.bbmt.2019.07.004

Guillermo CL et al (2007) Clinical features and survival of Chilean patients with multiple myeloma. Rev Med Chil 135(9):1111-7. https://doi: 10.4067/s0034-98872007000900003

He JS et al (2021) Multiple Extramedullary-Bone Related and/or Extramedullary Extraosseous Are Independent Poor Prognostic Factors in Patients With Newly Diagnosed Multiple Myeloma. Front Oncol 11:668099. https://doi: 10.3389/fonc.2021.668099

Ikeda T et al (2019) Comparison between autologous and allogeneic stem cell transplantation as salvage therapy for multiplemyeloma relapsing/progressing after autologous stem cell transplantation. Hematol Oncol 37(5):586-594. https://doi: 10.1002/hon.2688

Jagosky MH, Usmani SZ (2020) Extramedullary Disease in Multiple Myeloma. Curr Hematol Malig Rep 15(2):62-71. https://doi: 10.1007/s11899-020-00568-3.

Jayakrishnan TT et al (2021) Disparities in the enrollment to systemic therapy and survival for patients with multiple myeloma. Hematol Oncol Stem Cell Ther 14(3):218-230. https://doi: 10.1016/j.hemonc.2020.09.005

Kumar S et al (2016) International Myeloma Working Group consensus criteria for response and minimal residual disease assessment in multiple myeloma. Lancet Oncol 17(8): e328-

e346. https://doi:10.1016/S1470-2045(16)30206-6

Kumar SK et al (2020). Carfilzomib or bortezomib in combination with lenalidomide and dexamethasone for patients with newly diagnosed multiple myeloma without intention for immediate autologous stem-cell transplantation (ENDURANCE): a multicentre, open-label, phase 3, randomised, controlled trial. Lancet Oncol 21(10):1317-1330. https://doi: 10.1016/S1470-2045(20)30452-6

Kumar SK et al (2017) Multiple myeloma. Nat Rev Dis Primers 3:17046. https://doi: 10.1038/nrdp.2017.46

Laura R et al (2006) Bortezomib: an effective agent in extramedullary disease in multiple myeloma. Eur $\mathrm{J}$ Haematol 76(5): 405-408. https://doi: 10.1111/j.0902-4441.2005.t01-1-EJH2

462.x 
Liu Y et al (2020) Genetic Basis of Extramedullary Plasmablastic Transformation of Multiple Myeloma. Am J Surg Pathol 44(6):838-848. https://doi: 10.1097/PAS.0000000000001459

Montefusco V et al (2019) Outcome of Paraosseous Extra-Medullary Disease in Newly Diagnosed Multiple Myeloma Patients Treated With New Drugs. Haematologica 105(1):193-200. https://doi:

10.3324/haematol.2019.219139

O'Donnell EK et al (2018) A phase 2 study of modified lenalidomide, bortezomib and dexamethasone in transplant-ineligible multiple myeloma. Br J Haematol 182(2):222-230. https://doi: 10.1111/bjh.15261

Rajkumar SV et al (2014) International Myeloma Working Group updated criteria for the diagnosis of multiple myeloma. Lancet Oncol 15(12):e538-48. https://doi: 10.1016/S1470-20

$45(14) 70442-5$

Rosiñol L et al (2004). Extramedullary multiple myeloma escapes the effect of thalidomide. Haematologica $89(7): 832-836$.

Tian C et al (2018). Clinical characteristics and prognosis of multiple myeloma with bone-related extramedullary disease at diagnosis. Biosci Rep 38(3):BSR20171697. https://doi: 10.1042/BSR20171697

Touzeau C, Moreau P (2016) How I treat extramedullary myeloma. Blood 127:971-6.

Varettoni M et al ( 2010) Incidence, presenting features and outcome of extramedullary disease in multiple myeloma: a longitudinal study on 1003 consecutive patients. Ann Oncol 21(2):325-30. https://doi: 10.1093/annonc/mdp329.

Wang J et al (2020). Clinical characteristics and outcomes of patients newly diagnosed with multiple myeloma with extramedullary disease. Zhonghua Xue Ye Xue Za Zhi 41(10):822-828. https://doi: 10.3760/cma.j.issn.0253-2727.2020.10.006

Zhang Y et al (2019) Prognostic Nomogram for the Overall Survival of Patients with Newly Diagnosed Multiple Myeloma. Biomed Res Int 5652935. https://doi: 10.1155/2019/5652935

\section{Figures}



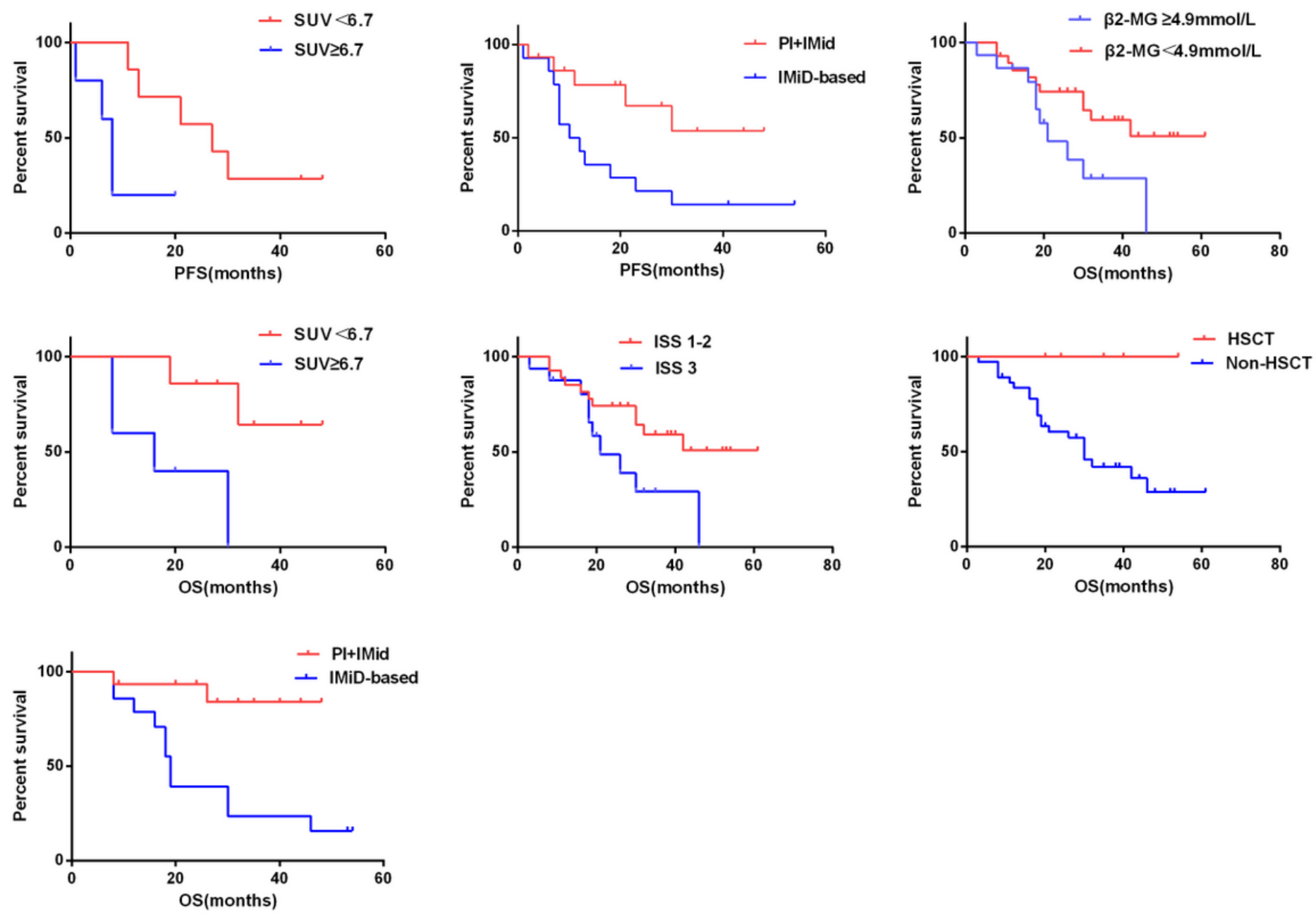

\section{Figure 1}

The survival of patients extramedullary multiple myeloma patients with single site invasion Among 43 patients with single site invasion, patients with SUVmax $\geq 6.7$ had significantly shorter $P F S(P<0.05)$. Among these patients, patients with $\beta 2$-microglobulin $\geq 4.9 \mathrm{~g} / \mathrm{L}$, SUVmax $\geq 6.7$, ISS stage 3 had poor OS $(P<0.05)$. PI combined with IMiD regimen was superior to IMiD in improving PFS and OS in patients with single site invasion, and HSCT was also associated with improved OS $(\mathrm{P}<0.05)$ 

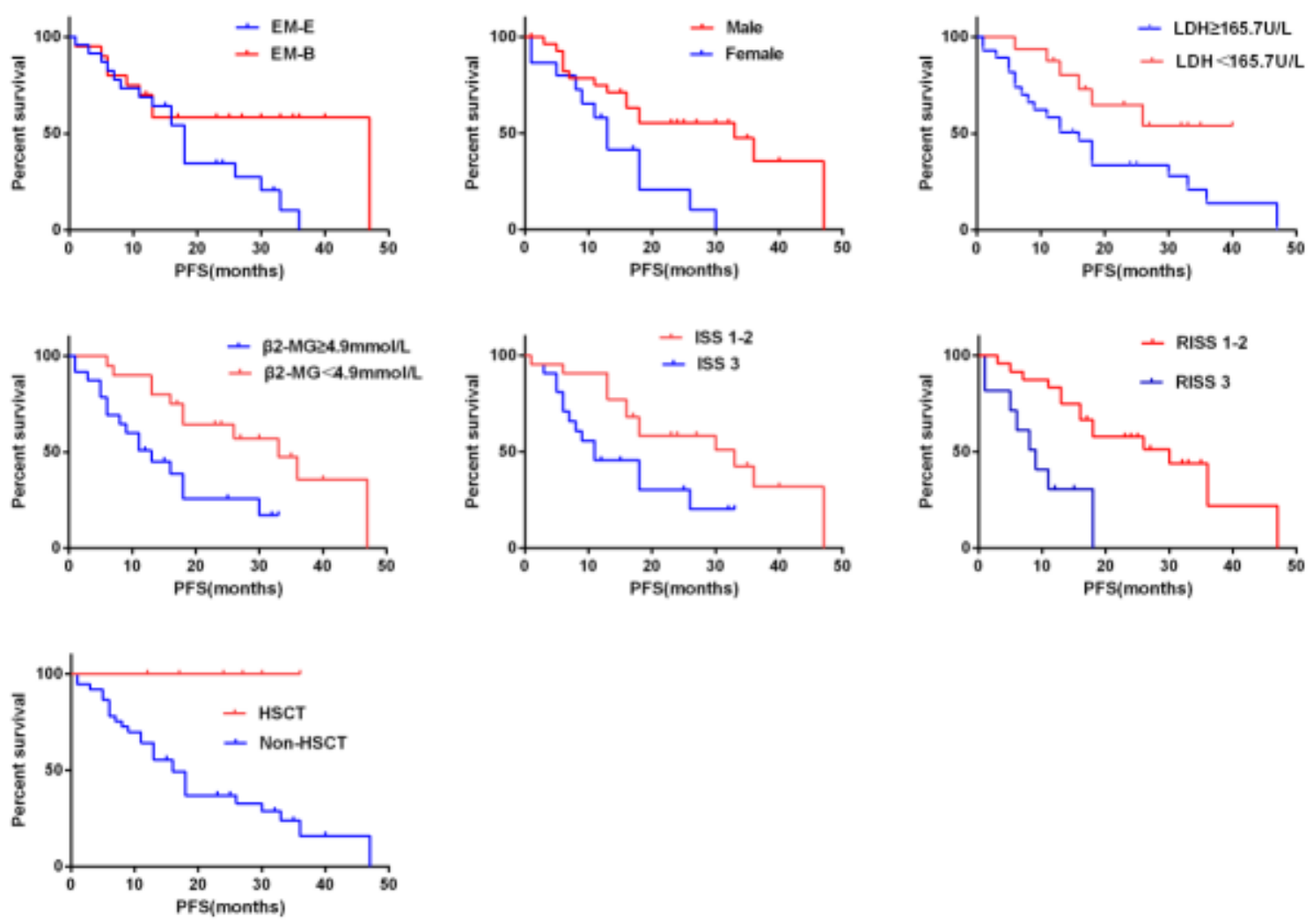

Figure 2

The progression free survival of patients extramedullary multiple myeloma patients with multiple sites invasion Among 47 patients with multiple sites invasion, patients with extramedullary extraosseous, female gender, $\beta 2$-microglobulin $\geq 4.9 \mathrm{~g} / \mathrm{L}, \mathrm{LDH} \geq 165.7 \mathrm{U} / \mathrm{L}$, ISS stage 3 and RISS stage 3 had significantly shorter PFS $(P<0.05)$. HSCT was also associated with improved PFS $(P<0.05)$. 

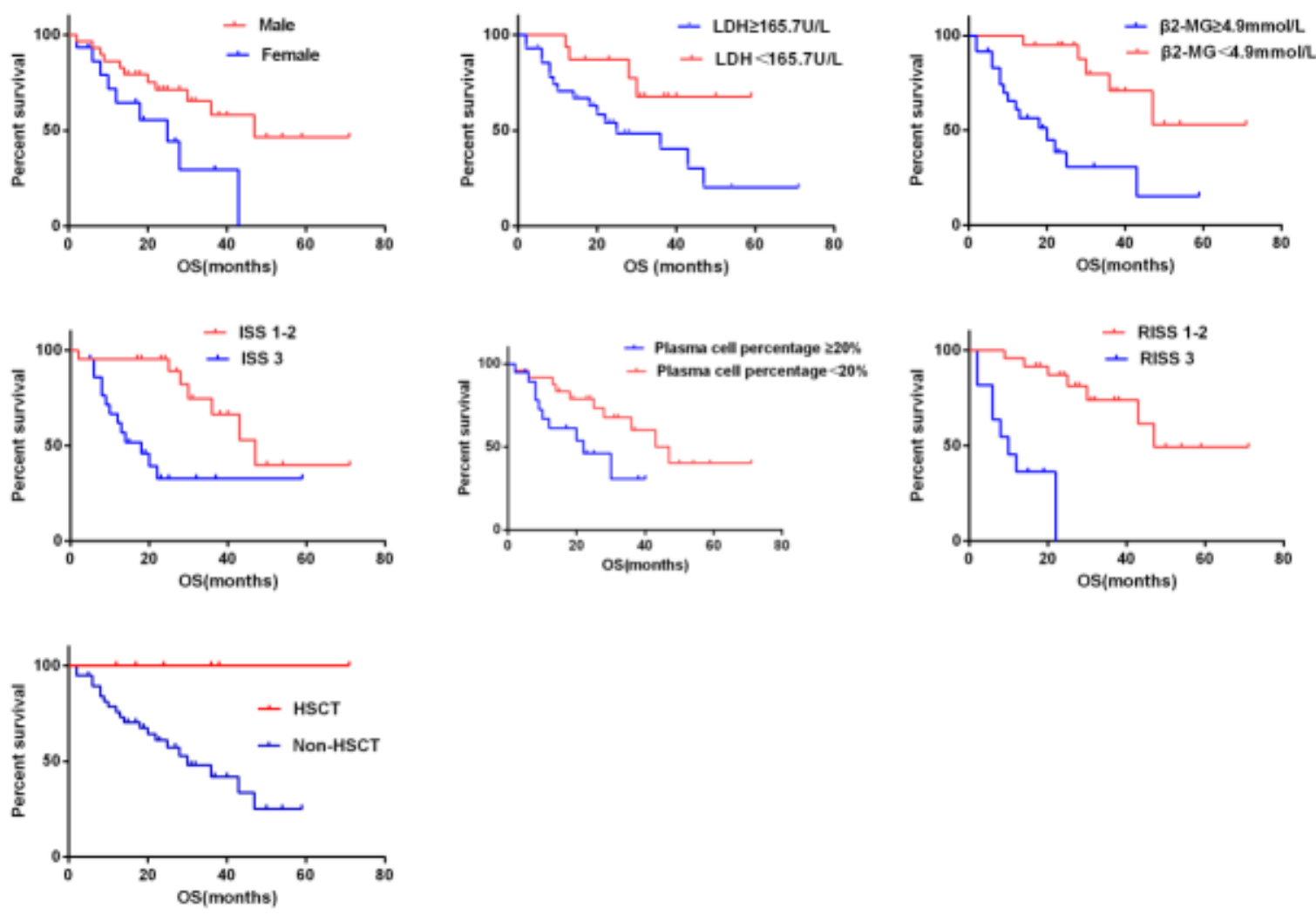

Figure 3

The overall survival of patients extramedullary multiple myeloma patients with multiple sites invasion Among 47 patients with multiple sites invasion, patients with female gender, $\beta 2$-microglobulin $\geq 4.9 \mathrm{~g} / \mathrm{L}$, $\mathrm{LDH} \geq 165.7 \mathrm{U} / \mathrm{L}$, plasma cell percentage $\geq 20 \%$, ISS stage 3 and RISS stage 3 also had poor OS $(P<0.05)$. HSCT was also associated with improved OS $(P<0.05)$. 